\title{
DETERMINATION OF THE OPTIMAL ENERGY DENOMINATOR SHIFT PARAMETER OF KRb ELECTRONIC STATES IN QUANTUM CHEMICAL COMPUTATIONS USING PERTURBATION THEORY
}

\author{
M. B. Shundalau ${ }^{*}$ and A. A. Minko
}

UDC 535.34

The influence of the energy denominator shift (EDS) parameter and the quantitative and qualitative compositions of electronic states included in $\operatorname{CASSCF}(2,14) / X M C Q D P T 2$ ab initio calculations of the ground state equilibrium internuclear distance and dissociation energy of polar KRb was determined.

Keywords: polar diatomic molecule, KRb, quantum chemical computation, electronic states, perturbation theory.

Introduction. Polar diatomic molecules (atomic pairs) consisting of different alkali metals ( $\mathrm{KRb}, \mathrm{KCs}, \mathrm{RbCs}$, etc.) are under intense experimental and theoretical scrutiny $[1,2]$ due to their ability to be used in quantum computers, to check the fundamental theory of the electron electric dipole moment, to carry out controlled chemical reactions at extremely low temperatures, etc. [3, 4]. Molecules in the ground rovibronic state must be produced in order to utilize these capabilities. One method for accomplishing this consists of converting polar diatomic molecules into the ground rovibronic state via initial optical excitation into higher lying rovibronic states with potential energy functions (PEFs) depending specifically on the internuclear distance. In this instance, the exact PEFs of the combining electronic states and the energy-spectral and dynamic characteristics of their vibronic subsystems must be known for highly effective excitation and subsequent conversion of the molecular system into the ground state.

Exact experimental PEF states are constructed by analyzing and interpreting high-resolution rovibronic spectra and are based mainly on potential curves obtained from ab initio calculations. Furthermore, ab initio calculations of a system of electronic states allow the whole required set of energy, spectral, and other characteristics of the rovibronic states to be computed. Quantum-chemical calculations (provided they are spectroscopically accurate) of the electronic structure and modeling of the energy-spectral characteristics of the electronic states of molecules including heavy alkali metals are nontrivial problems. Relativistic corrections for inner electrons, static and dynamic electron-correlation energy components, a large number of excited configurations, and other factors must be considered.

The diatomic polar $\mathrm{KRb}$ molecule is a typical example of the aforementioned compounds. The energy of the $\mathrm{KRb}$ ground state $X^{1} \Sigma^{+}$as a function of the internuclear distance was studied systematically [5]. The spectroscopic parameters of the $X^{1} \Sigma^{+}$state (equilibrium internuclear distance $R_{e}$, asymptotic limit $D_{e}$, etc.) were obtained and the experimental PEF was constructed by analyzing ultrahigh-resolution laser-induced fluorescence spectra. Ab initio calculations of the electronic states of $\mathrm{KRb}$ and other similar molecules are traditionally performed using complete active space self-consistent field/multireference configuration interaction (CASSCF/MRCI) [6-8]. This approach to multi-configurational problems enables both the static component of the electronic correlation energy (CASSCF) and its dynamic part (MRCI) to be considered. Both theory levels that are used sequentially in this instance are based on the variation principle. The calculations reproduced sufficiently successfully the experimental equilibrium internuclear distance $R_{e}(4.06770 \AA$ [5]) of ground electronic state $X^{1} \Sigma^{+}: 4.055$ [6], 4.09 [7], and $4.095 \AA$ [8]. Moreover, the calculations predicted substantially reduced dissociation energies $D_{e}$ of the ground-state molecule (4106 [6], 4114 [7], and $4130 \mathrm{~cm}^{-1}$ [8]; experimental value $4217.815 \mathrm{~cm}^{-1}$ [5]). A new approach to multi-state multi-reference perturbation theory (MS-MRPT) that was recently developed [9] and was called extended multi-configuration quasi-degenerate perturbation theory (XMCQDPT) enables these problems to be examined using an alternative approach based on perturbation theory and not the variation principle.

*To whom correspondence should be addressed.

Belarusian State University, 4 Nezavisimost' Ave., Minsk, 220030, Belarus; e-mail: shundalov@bsu.by. Translated from Zhurnal Prikladnoi Spektroskopii, Vol. 82, No. 6, pp. 821-825, November-December, 2015. Original article submitted April 3, 2015. 

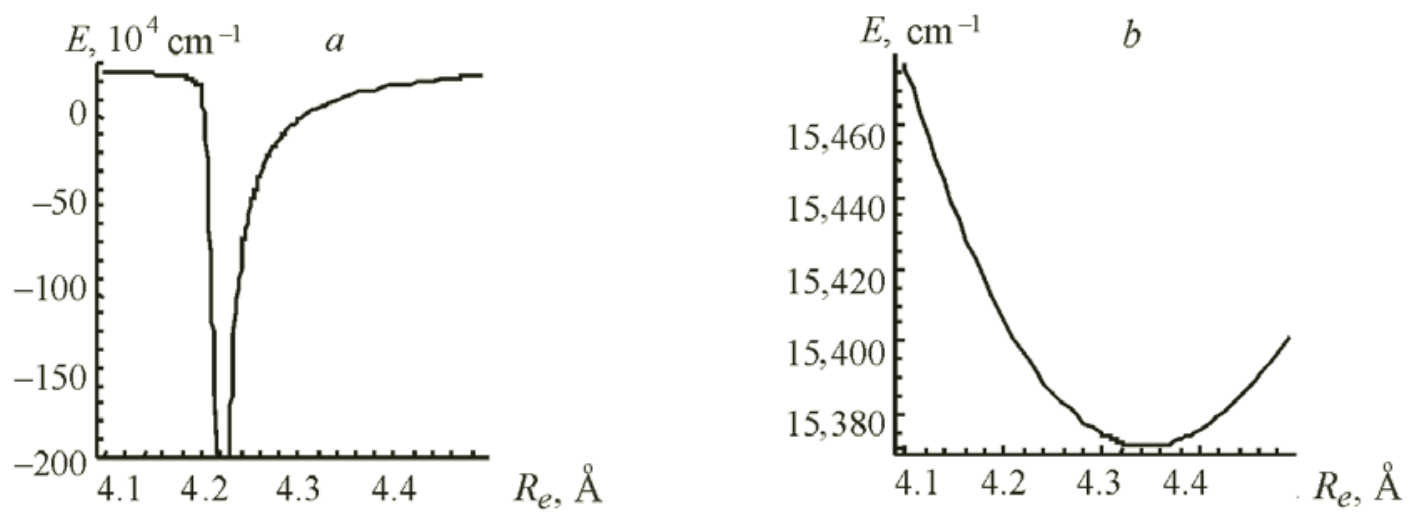

Fig. 1. Portion of the potential energy function from $\operatorname{CASSCF}(2,14) / \mathrm{XMCQDPT} 2$ calculations of the $\mathrm{KRb} 1^{1} \Pi$ term with $b=0.0$ (a) and 0.001 (b); the minimum of groundstate term $X^{1} \Sigma^{+}$corresponds to zero potential energy.

The principal goal of the present work was to find the optimal parameters for non-empirical quantum-chemical CASSCF/MRPT calculations of the electronic state characteristics of polar diatomic molecules including heavy alkali metals using $\mathrm{KRb}$ as an example.

Calculations. An important feature of MRPT-level theory is the ability to vary the number of states (and their qualitative composition) considered in the perturbation theory method in order to achieve the best agreement with the experimental data and to use the results further for predictions. Another specific feature of MRPT-based quantum-chemical calculations is the appearance of so-called intruder states [10]. Such artefacts are generated because of the truncating feature of quantum-chemical calculations in terms of perturbation theory for a multi-reference system. A second-order correction to the energy of state $\alpha$ generated by its coupling with state $s$ is [10]:

$$
E_{\alpha, s}^{(2)}=-\frac{|\langle s|H| \alpha\rangle|^{2}}{E_{s}^{(0)}-E_{\alpha}^{(0)}},
$$

where $H$ is the unperturbed Hamiltonian; $E_{s}^{(0)}$ and $E_{\alpha}^{(0)}$, the zero-order energies of states $s$ and $\alpha$.

The situation $E_{s}^{(0)} \approx E_{\alpha}^{(0)}$ is possible for quasi-degenerate states (which is often encountered for excited terms). According to Eq. (1), the second-order correction to the energy approaches infinity and a break occurs in the PEF profile (Fig. 1a). A non-zero correction $\Delta_{s}$ to the energy of state $s$ (energy denominator shift, EDS) is introduced in order to avoid the non-physical behavior of the PEF [10]:

$$
\tilde{E}_{s}^{(0)}=E_{s}^{(0)}+\Delta_{s},
$$

which is usually written

$$
\Delta_{S}=b /\left(E_{s}^{(0)}-E_{\alpha}^{(0)}\right),
$$

where $b$ is a certain non-zero parameter. The difference $E_{s}^{(0)}-E_{\alpha}^{(0)}$ is never equal to zero and the PEF does not develop breaks if the energy of the perturbing state is redefined in this manner (Fig. 1b). The value 0.02 for parameter $b$ is usually recommended in user guides for quantum-chemical programs. It is assumed that it has little effect on the characteristics of the calculated states of polyatomic molecules. However, it was shown [11] that parameter $b$ for diatomic molecules has a significant effect on the accuracy of the reproduced experimental PEF characteristics. Thus, calculations in terms of MRPT [11] using the GAMESS software [12] agreed best with the experimental data for a whole series of diatomic molecules including atoms in the first two rows of the Periodic Table $\left(\mathrm{CH}, \mathrm{NH}, \mathrm{CO}, \mathrm{O}_{2}\right.$, etc.) for $b \sim 0.3$, which was an order of magnitude greater than the recommended value [12]. 


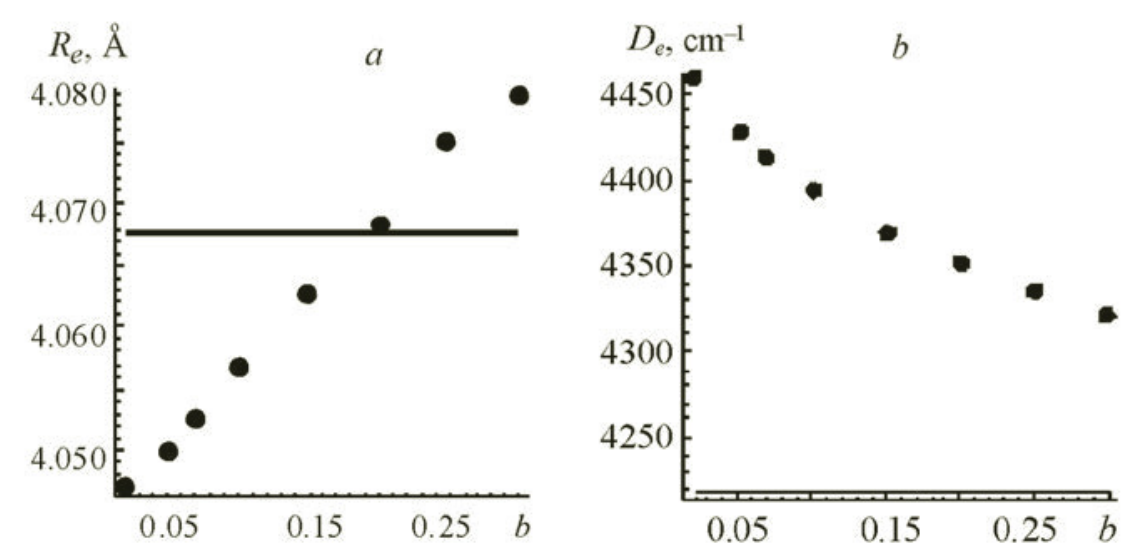

Fig. 2. Equilibrium internuclear distance $R_{e}$ (a) and dissociation energy $D_{e}$ (b) of the $\mathrm{KRb}$ ground-state term as a function of EDS parameter $b$ from CASSCF(2,14)/IMCQDPT2 calculations including 16 states in the perturbation theory method; lines are experimental values [5].
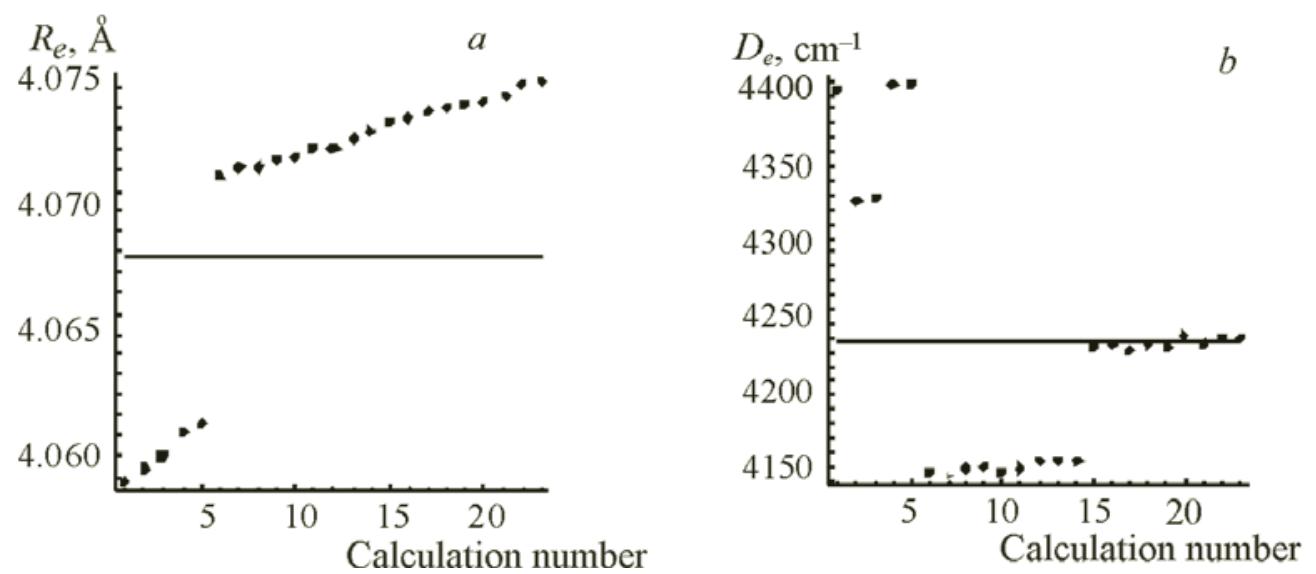

Fig. 3. Equilibrium internuclear distance $R_{e}$ (a) and dissociation energy $D_{e}$ (b) of the $\mathrm{KRb}$ ground-state term as a function of the quantitative and qualitative composition of states in the perturbation theory method; lines are experimental values [5].

Energies of states at several reference points were calculated in terms of perturbation theory for various $b$ values $(0-0.3)$ and a fixed number (16) of states in order to find the optimal values of $b$ for the examined problem. The calculations used the Firefly applied quantum-chemical software [13] and Stuttgart RSC ECP (effective core potential) basis sets ECP10MDF and ECP28MDF [14] for K and Rb atoms.

CASSCF calculations with a density-matrix averaging procedure over 13 states were performed for two active electrons involving 14 active orbitals of a molecule with trivial symmetry $\left(\mathrm{C}_{1}\right)$. In this instance, all examined electronic states (both non-degenerate $\Sigma^{+}$and doubly degenerate $\Pi$ and $\Delta$ ) belonged to the same symmetry type $(A)$ and were partially mixed in the averaging procedure. This situation described more realistically the electronic structure of the molecular system. Then, XMCQDPT2 calculations were performed [9]. The electron-correlation energy was considered for all eight doubly occupied orbitals in the ground configuration.

Results and Discussion. Figure 2 shows results from the calculations and indicates that the spectroscopic parameters of $\mathrm{KRb}$ depended strongly on $b$. The function was smooth for realistic $b$ values. The equilibrium internuclear distance increased with increasing $b$ whereas the dissociation energy decreased. However, whereas the optimal value of $b$ for $R_{e}$ lay close to 0.20 (with a certain fixed number of states in the XMCQDPT2 procedure), at which the calculation reproduced the experimental $R_{e}$, the optimal value of $b$ for $D_{e}$, if it existed at all, was far beyond the limits of its realistic values. 
The optimal quantitative and qualitative compositions of electronic states included in the XMCQDPT2 procedure were assessed for a more accurate reproduction of the $\mathrm{KRb}$ experimental spectroscopic parameters. The number of states varied from 7 to 16 . The qualitative composition included $\Sigma^{+}$-, $\Pi$-, and $\Delta$-states. The calculations used $b=0.10$. The calculations were ordered according to increasing calculated $R_{e}$ (Fig. 3). The initial set (calculation 14) consisted of 7 lower singlet states of $\mathrm{KRb}$ (ground $X^{1} \Sigma^{+}$and excited $2^{1} \Sigma^{+}, 3^{1} \Sigma^{+}, 1^{1} \Pi$, and $2^{1} \Pi$ ). All other calculations included necessarily this set of states and also several others. Calculation 1 included the seven initial states in addition to $4^{1} \Sigma^{+}, 5^{1} \Sigma^{+}, 6^{1} \Sigma^{+}, 1^{1} \Delta, 3^{1} \Pi$, and $4^{1} \Pi$ (16 total states); calculation 2 (here and further, only states in addition to the seven initial ones are given), $4^{1} \Sigma^{+}$and $6^{1} \Sigma^{+}$(9 states); calculation $3,4^{1} \Sigma^{+}(8$ states $)$; calculation $4,4^{1} \Sigma^{+}, 5^{1} \Sigma^{+}$, and $6^{1} \Sigma^{+}(10$ states $)$; calculation $5,4^{1} \Sigma^{+}$and $5^{1} \Sigma^{+}$(9 states); calculation $6,1^{1} \Delta, 3^{1} \Pi$, and $4^{1} \Pi\left(13\right.$ states); calculation $7,1^{1} \Delta$ and $3^{1} \Pi$ (11 states); calculation $8,1^{1} \Delta$ and $4^{1} \Pi$ (11 states); calculation $9,3^{1} \Pi$ and $4^{1} \Pi$ (11 states); calculation $10,1^{1} \Delta$ (9 states); calculation $11,3^{1} \Pi$ (9 states); calculation 12 , $4^{1} \Pi$ (9 states); calculation $13,6^{1} \Sigma^{+}$(8 states); calculation $15,5^{1} \Sigma^{+}, 6^{1} \Sigma^{+}, 1^{1} \Delta, 3^{1} \Pi$, and $4^{1} \Pi(15$ states $)$; calculation $16,5^{1} \Sigma^{+}$, $6^{1} \Sigma^{+}, 1^{1} \Delta$, and $4^{1} \Pi$ (13 states); calculation $17,5^{1} \Sigma^{+}, 6^{1} \Sigma^{+}, 1^{1} \Delta$, and $4^{1} \Pi$ (13 states); calculation $18,5^{1} \Sigma^{+}, 6^{1} \Sigma^{+}, 3^{1} \Pi$, and $4^{1} \Pi$ (13 states); calculation $19,5^{1} \Sigma^{+}, 6^{1} \Sigma^{+}$, and $1^{1} \Delta$ (11 states); calculation $20,5^{1} \Sigma^{+}, 6^{1} \Sigma^{+}$, and $4^{1} \Pi$ (11 states); calculation $21,5^{1} \Sigma^{+}, 6^{1} \Sigma^{+}$, and $3^{1} \Pi$ (11 states); calculation $22,5^{1} \Sigma^{+}$and $6^{1} \Sigma^{+}$(9 states); and calculation $23,5^{1} \Sigma^{+}$(8 states).

Thus, inclusion of $4^{1} \Sigma^{+}$and $5^{1} \Sigma^{+}$states in perturbation-theory calculations played a decisive role in reproducing the experimental spectroscopic parameters of the KRb ground state because calculations $1-5$, which predicted a reduced equilibrium internuclear distance and substantially increased dissociation energy, all included the $4^{1} \Sigma^{+}$state. Calculations 15-23, which agreed best with the experimental data, contained the $5^{1} \Sigma^{+}$state. Consideration of the $6^{1} \Sigma^{+}$state and doubly degenerate $\Pi$ - and $\Delta$-states did not noticeably affect the spectroscopic parameters of the $X^{1} \Sigma^{+}$ground state. The non-zero contributions of $\Pi$ - and $\Delta$-states were due to partial mixing of non-degenerate $\Sigma^{+}$and doubly degenerate $\Pi$ - and $\Delta$-states during the averaging. It is also noteworthy that dissociation energies obtained in calculations 6-14 agreed with CASSCF/ MRCI calculations [6-8].

Conclusions. The optimal parameters for XMCQDPT2-level of theory calculations could reproduce characteristics of the $\mathrm{KRb}$ electronic ground state with close to spectroscopic accuracy. The obtained features could be used for quantumchemical calculations of the electronic terms of diatomic molecules with electronic structures similar to that of KRb.

Acknowledgments. The work was sponsored by the Republic of Belarus State Committee for Science and Technology (Project No. F14LAT-060).

\section{REFERENCES}

1. O. Dulieu and C. Gabbanini, Rep. Prog. Phys., 72, 086401 (2009).

2. R. V. Krems, W. C. Stwalley, and B. Friedrich (Eds.), Cold Molecules: Theory, Experiment, Applications, CRC Press, Boca Raton (2009).

3. J. M. Hutson and P. Soldan, Int. Rev. Phys. Chem., 25, 497-526 (2006).

4. G. Qiemener and P. S. Julienne, Chem. Rev., 112, 4949-5011 (2012).

5. A. Pashov, O. Docenko, M. Tamanis, R. Ferber, H. Knockel, and E. Tiemann, Phys. Rev. A: At., Mol., Opt. Phys., 76, 022511 (2007).

6. S. Rousseau, A. R. Allouche, and M. Aubert-Frecon, J. Mol. Spectrosc., 203, 235-243 (2000).

7. S. J. Park, Y. J. Choi, Y. S. Lee, and G.-H. Jeung, Chem. Phys., 257, 135-145 (2000).

8. K. Chen, C.-L. Yang, M.-S. Wang, X.-G. Ma, and W.-W. Liu, Spectrochim. Acta, Part A, 99, 57-61 (2012).

9. A. A. Granovsky, J. Chem. Phys., 134, 214113 (2011).

10. H. A. Witek, Y.-K. Choe, J. P. Finley, and K. Hirao, J. Comput. Chem., 23, 957-965 (2002).

11. S.-W. Chang and H. A. Witek, J. Chem. Theory Comput., 8, 4053-4061 (2012).

12. http://www.msg.ameslab.gov/GAMESS/GAMESS.html

13. A. A. Granovsky, Firefly version 8 ; http://classic.chem.msu.su/gran/firefly/index.html

14. I. S. Lim, P. Schwerdtfeger, B. Metz, and H. Stoll, J. Chem. Phys., 122, 104103 (2005). 